\title{
1 Rapid evolution promotes fluctuation-dependent species coexistence
}

Last revision: December 11, 2020

$4 \quad{ }^{1}$ School of Biological Sciences, The University of Queensland, St. Lucia, Brisbane, Queensland

5 4072, Australia, ${ }^{2}$ Department of International Health and Medical Anthropology, Institute of

8 Short title: Eco-evolutionary relative nonlinearity

9 Keywords: Adaptive foraging, Armstrong-McGehee mechanism, breeding suppression,

10 competition, dormancy, eco-evolutionary dynamics, fitness gradient, gleaner-opportunist trade-

11 off, phenotypic plasticity, relative nonlinearity 


\section{Abstract}

13 Recent studies have demonstrated that rapid contemporary evolution can play a significant role in

14 regulating population dynamics on ecological timescales. Here we identify a previously

15 unrecognized mode by which rapid evolution can promote species coexistence via temporal

16 fluctuations and a trade-off between competitive ability and the speed of adaptive evolution. We

17 show that this interaction between rapid evolution and temporal fluctuations not only increases

18 the range of coexistence conditions under a gleaner-opportunist trade-off (i.e., low minimum

19 resource requirement $\left[R^{*}\right]$ vs. high maximum growth rate), but also yields stable coexistence in

20 the absence of a classical gleaner-opportunist trade-off. Given the propensity for both oscillatory

21 dynamics and divergent rates of adaptation (including rapid evolution and phenotypic plasticity)

22 in the real world, we argue that this expansion of fluctuation-dependent coexistence theory

23 provides an important overlooked solution to the so-called 'paradox of the plankton'. 


\section{Introduction}

25 A wealth of empirical evidence has accumulated over the last few decades indicating that rapid

26 contemporary evolution on ecological timescales (Hendry 2016) can be essential for

27 understanding population dynamics (Yoshida et al. 2003; Bell 2017). More recently, evidence has

28 begun to emerge that rapid evolution can also be a significant driver of community dynamics

29 amongst competing species, and in particular play an important role in regulating species

30 coexistence (Lankau 2011; Vasseur et al. 2011; Mougi 2013; Hiltunen et al. 2017; Wittmann \&

31 Fukami 2018; Hart et al. 2019; Germain et al. 2020; van Velzen 2020; Yamamichi et al. 2020).

32 To date, however, rapid evolution has only been shown to promote species coexistence when

33 there is either a trade-off between traits optimal for intraspecific and interspecific competition

34 (Lankau 2011; Vasseur et al. 2011; Mougi 2013; Wittmann \& Fukami 2018; Yamamichi et al.

35 2020) or fine-tuning of prey defenses and predator foraging efforts (Kondoh 2003; van Velzen

36 2020). Here we provide a previously unrecognized pathway for rapid evolution to promote

37 coexistence via temporal fluctuations: differences in the rate of adaptation to fluctuating

38 environments. Specifically, using model simulations, we show that rapidly evolving and non-

39 evolving consumers can stably coexist even when they compete for a single resource. 
41 Understanding how species coexist in nature has been a central topic in community ecology over

42 the last century (Hutchinson 1961; Wilson 1990). G. E. Hutchinson (1961) famously referred to

43 the problem as "the paradox of the plankton" on account of the seemingly impossible challenge

44 of explaining the extraordinary diversity of oceanic plankton (Hutchinson 1961). Independent of

45 emerging interest into rapid evolution, a growing body of coexistence research has suggested that

46 temporal fluctuations can promote diversity via two broad classes of mechanisms, the temporal

47 storage effect and relative nonlinearity (Chesson 1994, 2000; Yuan \& Chesson 2015; Barabás et

48 al. 2018; Ellner et al. 2019). Although greater attention has been given to the temporal storage

49 effect (Adler et al. 2006; Angert et al. 2009; Letten et al. 2018; Hallett et al. 2019; Zepeda \&

50 Martorell 2019), recent studies have shown that relative nonlinearity can be more important than

51 previously thought for promoting coexistence (Letten et al. 2018; Hallett et al. 2019; Zepeda \&

52 Martorell 2019). In this paper, we introduce rapid evolution as a previously neglected driver of

53 relative nonlinearity.

55 Relative nonlinearity promotes coexistence when one species benefits from fluctuations in the 
56 intensity of competition (fluctuation-assisted) while the other species dominates in the absence of

57 fluctuations (fluctuation-impeded). In addition to their responses to fluctuations, for the two

58 competing species to coexist, they need different impacts on fluctuations. If the fluctuation-

59 assisted species reduces the amplitude of fluctuations (by "consuming" the variance in the

60 intensity of competition (Levins 1979)) while the fluctuation-impeded species increases it, each

61 species produces a favorable environment for the competitor, resulting in negative frequency-

62 dependence in community dynamics (Hsu et al. 1978; Armstrong \& McGehee 1980) (Fig. 1a-b).

64 As the name suggests, two species may coexist via relative nonlinearity when consumer

65 population growth responses to a limiting resource differ in their degree of nonlinearity (Fig. 1c-

66 d) (Hsu et al. 1978; Armstrong \& McGehee 1980; Abrams \& Holt 2002; Abrams et al. 2003;

67 Xiao \& Fussmann 2013). The differential responses to, and impacts on, fluctuations are most

68 conspicuous when competing consumers exhibit intermittent cycles (Fig. 1d), where the linear,

69 competitively inferior species at equilibrium (with larger $R^{*}$ ), increases in abundance when the

70 system oscillates but simultaneously dampens the consumer-resource cycles induced by the

71 competitively superior species (with smaller $R^{*}$ ). In contrast, the nonlinear, competitively 
72 superior species generates fluctuations but increases in abundance when the system is stable (Fig.

73 1d). The species with the lowest $R^{*}$ value would outcompete its competitor under a stable

74 equilibrium, but the linear species benefits from fluctuating environments in competition due to

75 nonlinear averaging of the low $R^{*}$ species' per capita growth response (Jensen's inequality). The

76 situation in Fig. 1c is called a gleaner-opportunist trade-off, where species 1 has the lower $R^{*}$

77 whereas species 2 has the higher maximum growth rate (Grover 1990). Because this criterion is

78 sometimes perceived as being highly restrictive, relative nonlinearity has typically been assumed

79 to play a minor role in real systems (Adler et al. 2006; Angert et al. 2009; Letten et al. 2018;

80 Hallett et al. 2019; Zepeda \& Martorell 2019). By demonstrating how rapid evolution can relax

81 these restrictions and significantly broaden opportunities for coexistence even without the

82 classical gleaner-opportunist trade-off, we argue that relative nonlinearity may operate much

83 more widely in nature than previously thought.

\section{METHODS}

86 The basis for the mechanism presented here is that rapid evolution can enable populations to

87 adapt to fluctuating environments (response) and to stabilize temporal fluctuations (impact) 
88 (Cortez \& Patel 2017). Thus, even when two species with fixed functional responses cannot

89 coexist, they may have different responses to, and impacts on, temporal fluctuations, facilitating

90 coexistence. We consider a model of two consumers with saturating consumer functional

91 responses competing for a single logistically growing resource (Rosenzweig \& MacArthur 1963;

92 Hsu et al. 1978; Xiao \& Fussmann 2013). In our model, the dynamics of the two consumers, $N_{1}$

93 and $N_{2}$, competing for a single resource, $R$, with quantitative traits, $x_{1}$ and $x_{2}$ is represented by

$$
\begin{aligned}
& \frac{d R}{d t}=R\left[r\left(1-\frac{R}{K}\right)-\sum_{j=1}^{2} \frac{a_{j}\left(x_{j}\right) N_{j}}{1+h_{j} R}\right], \\
& \frac{d N_{i}}{d t}=N_{i}\left[\frac{c_{i} a_{i}\left(x_{i}\right) R}{1+h_{i} R}-d_{i}\left(x_{i}\right)\right], \quad(i=1,2), \\
& \frac{d x_{i}}{d t}=V_{i} \frac{\partial}{\partial x_{i}}\left(\frac{1}{N_{i}} \frac{d N_{i}}{d t}\right), \quad(i=1,2),
\end{aligned}
$$

97 where $r$ is the intrinsic growth rate, $K$ is the carrying capacity, $a_{i}$ is the parameter that increases

98 the consumption rate as a whole (including the slope and maximum value), $b_{i}$ is the parameter

99 that reduces the maximum consumption rate, $c_{i}$ is the conversion efficiency, $d_{i}$ is the consumer

100 mortality, and $V_{i}$ is additive genetic variance of the trait $x_{i}(i=1,2)$. 
102 We assume a trade-off between parameters for the consumption rate, $a_{i}$, and mortality rate, $d_{i}$,

103 such that increasing the consumption rate increase mortality rate in an accelerating manner $\left(a_{i}\left(x_{i}\right)\right.$

$\left.104=\alpha_{i} x_{i}, d_{i}\left(x_{i}\right)=\delta_{0 i}+\delta_{1 i} x_{i}+\delta_{2 i} x_{i}^{2}\right)$. This occurs due to, for example, a foraging-predation risk trade-

105 off (Verdolin 2006) where predators have Holling type III functional responses. In addition, we

106 assume the trait changes along the fitness gradient (Abrams 2001; Cortez \& Patel 2017). Thus,

107 trait dynamics can be represented by

108

$$
\frac{d x_{i}}{d t}=V_{i}\left(\frac{c_{i} \alpha_{i} R}{1+h_{i} R}-\delta_{1 i}-2 \delta_{2 i} x_{i}\right)
$$

111 and the optimal trait at a certain resource level is obtained by solving $d x_{i} / d t=0$, and it is given by

$112 x_{i}=\left(c_{i} \alpha_{i} R /\left(1+b_{i} R\right)-\delta_{1 i}\right) /\left(2 \delta_{2 i}\right)$. There is stabilizing (instead of disruptive) selection toward the

113 optimal trait as $\partial\left(d x_{i} / d t\right) / \partial x_{i}=-2 V_{i} \delta_{2 i}<0$. This indicates that the evolving consumer invests more

114 in resource uptake when resources are abundant, whereas it tries to reduce its mortality rate when

115 resources are scarce. Furthermore, by nondimensionalization, we can assume that $r=K=c_{i}=1$

116 without loss of generality (Appendix S1). 
118 As the resource exhibits logistic growth and consumers exploit the resource with a Holling type

119 II functional response, the system corresponds to the classical Rosenzweig-MacArthur model

120 when there is a single non-evolving consumer species (Rosenzweig \& MacArthur 1963). In the

121 absence of fluctuations, the consumer with the smaller resource requirement (i.e., smaller $R^{*}$ )

122 reduces the resource level to the point $\left(R_{i}^{*}=d_{i} /\left(a_{i} c_{i}-b_{i} d_{i}\right), i=1,2\right)$ where its competitor with a

123 larger $R^{*}$ cannot persist and is excluded. On the other hand, in the presence of endogenous limit

124 cycles (e.g., with larger $K$ values) the two consumers may coexist via relative nonlinearity. Given

125 Holling type II (concave) functional responses, population growth rates of the two species are

126 lowered by fluctuations, but the species with lower $R^{*}$ is more impeded by fluctuations in the

127 intensity in competition, thereby promoting coexistence (Hsu et al. 1978; Xiao \& Fussmann

128 2013).

129

130 Results

131 When we assume that a competitively superior species (with smaller $R^{*}$ at equilibrium) does not

132 evolve, whereas an evolving, competitively inferior (larger $R^{*}$ ) species with the optimum trait

133 either grows faster than the competitively superior species when the resource is abundant (Fig. 
134 2a) or scarce (Fig. 2b), coexistence is possible. Notably, our parameter settings do not satisfy

135 necessary conditions for coexistence via the classical gleaner-opportunist trade-off identified in

136 previous studies (Appendix S1, Fig. S1) (Hsu et al. 1978; Xiao \& Fussmann 2013) for any fixed

137 trait values. Thus, when the speed of evolution in the species that is competitively inferior at

138 equilibrium is slow, competitive exclusion ensues (Fig. S2). However, when evolution is faster,

139 coexistence is possible (Fig. 2). This occurs because relative to the non-evolving species, the

140 rapidly evolving species benefits from resource fluctuations, but at the same time rapid evolution

141 dampens the consumer-resource cycles (Appendix S2, Fig. S3) induced by the non-evolving

142 superior competitor, as can be clearly seen when the system cycles intermittently (Fig. 2f).

143 Therefore, rapid evolution works as a compensation mechanism for the species that would

144 otherwise be competitively inferior in a non-fluctuating system.

146 The underlying mechanism for coexistence (sensu Chesson 2000) can be understood by looking

147 at the very-rapid-evolution limit. When evolution is much faster than ecological processes, the

148 evolving species is always able to attain the optimal trait value that maximizes per-capita growth

149 rate for a given resource concentration. The effect of very rapid evolution is to change the 
150 effective functional responses of the evolving species from concave down (gray curves in Fig. 2a-

151 b) to concave up (especially when the limiting resource is scarce: orange curves in Fig. 2a-b) and

152 it is this change that allows coexistence by enabling the evolving species to benefit from

153 fluctuating environments in competition through nonlinear averaging. Although the realized per-

154 capita growth rates when evolution is not much faster than ecology are different from those based

155 on the optimal trait values due to the time lag in responses to changing environments (Fig. 2c-d,

156 Fig. S4), coexistence is still possible because of relative nonlinearity.

158 It is important to note that rapid evolution not only broadens the parameter conditions that allow

159 for coexistence via a so-called gleaner-opportunist trade-off (i.e., species 1 has a lower $R^{*}$

160 whereas species 2 has a higher maximum growth rate (Grover 1990): Fig. 2a, c, and e), but it also

161 yields stable coexistence under conditions previously thought to be incompatible with

162 coexistence (Fig. 3). More specifically, coexistence is still possible even in the absence of a

163 classical gleaner-opportunist trade-off, i.e., there is no trade-off between $R^{*}$ and maximum growth

164 rate, and the species with the smaller $R^{*}$ and the higher maximum growth rate (species 1 in Fig.

$1652 \mathrm{~b}, \mathrm{~d}$, and $\mathrm{f}$ ) is competitively impeded by fluctuations. Figure $3 \mathrm{~b}$ shows that coexistence is not 
166 possible when genetic variance is zero, but as the genetic variance of the inferior species

167 increases, the range of parameter values compatible with coexistence sharply increases. As such,

168 this relaxation of the perceived restrictions characterizing classical relative nonlinearity can

169 explain the coexistence of a much greater functional diversity of competing organisms than

170 would be possible in the absence of rapid evolution.

172 Discussion

173 Relative nonlinearity promotes species coexistence via fluctuations when (1) growth rates are

174 relatively nonlinear functions of the limiting factor in competition (in this case, a shared resource)

175 and (2) each species, when abundant, drives the amplitude of fluctuations in directions that favor

176 their competitors (Fig. 1) (Yuan \& Chesson 2015). Here we have shown that rapid evolution can

177 satisfy the above two conditions under conditions previously thought to be incompatible with

178 coexistence. Even without the classical gleaner-opportunist trade-off (Grover 1990), we show

179 that coexistence is possible via relative nonlinearity as the evolving consumer species benefits

180 from resource fluctuations in competition but stabilizes the consumer-resource cycles (Fig. 2-3). 
182 Although our focus here is a system with rapidly evolving consumers competing for a single

183 resource, a trade-off between competitive ability at equilibrium and the speed of adaptation may

184 promote coexistence via relative nonlinearity under various other scenarios. For example,

185 mechanisms of rapid adaptive trait changes include rapid evolution and phenotypic plasticity, and

186 some organisms plastically suppress foraging activity and reproduction when resources are scarce

187 (breeding suppression or dormancy), thereby stabilizing population dynamics (Nakazawa et al.

188 2009). This type of adaptive behavioral responses may also promote coexistence via relative

189 nonlinearity (Appendix S4, Fig. S6). Recently, Tan et al. (2020) showed that a competitively

190 inferior (larger $R^{*}$ ) consumer species with resource-dependent dormancy can coexist with a

191 superior species in consumer-resource cycles. Although the authors discussed their findings in the

192 context of game theory, our results indicate that they can also be explained as an outcome of

193 relative nonlinearity. In addition, phenotypic plasticity in defense traits against predation can

194 stabilize population dynamics (Yamamichi et al. 2019) and may also promote coexistence via

195 relative nonlinearity. When the limiting factor in competition is predation pressure instead of

196 resource abundance (i.e., apparent competition), species with higher predation tolerance $\left(P^{*}\right)$ will

197 persist (Holt 1977). However, coexistence is possible when species with lower predation 
198 tolerance stabilizes predator-prey cycles by inducible defenses (Appendix S5, Fig. S7). This

199 provides an explanation for previous simulation results demonstrating coexistence of three prey

200 types (asexually reproducing clones) with a shared predator and a shared resource (Yamamichi et

201 al. 2011). According to classical $R^{*}$ and $P^{*}$ theory, only two species (or asexual clones) can

202 coexist with a single predator and a single resource (Holt et al. 1994), but a third prey species

203 with inducible defense can coexist with two non-plastic (defended and undefended) prey by

204 relative nonlinearity. The destabilizing non-plastic prey have a competitive advantage when the

205 dynamics are stable, while the stabilizing plastic prey have an advantage under fluctuations.

207 While our model assumptions are quite general, further theoretical studies are necessary to

208 understand the generality and robustness of this novel mode of coexistence in ecosystems

209 including, for example, the shape of the trade-off between consumption and mortality (Appendix

210 S3, Fig. S5). Analyzing stochastic models will be particularly helpful to understand how low

211 genetic variance (and therefore slower rates of evolution) in small populations impedes

212 coexistence. It will be also valuable to conduct empirical studies that examine the effects of rapid

213 evolution on species coexistence via relative nonlinearity. In addition to meta-analyses on the 
214 trade-off between foraging and mortality (Verdolin 2006) and the trade-off between competitive

215 ability at equilibrium and the speed of adaptation, competition experiments will be valuable for

216 deepening our understandings. Previous studies on the consequences of rapid evolution have

217 typically used a single clone for competition experiments after long-term experimental evolution

218 (Hart et al. 2019). However, it is rapid evolution itself during competition experiments that can

219 promote coexistence via the mechanism outlined here, and a single clone cannot produce these

220 kinds of dynamics. Although it is possible to manipulate the amount of genetic variation by

221 changing the number of clones (Yoshida et al. 2003; Becks et al. 2010), we note that species with

222 two clones will not coexist with another species via relative nonlinearity (mathematically, it is

223 equivalent to three consumers competing for a single resource). In addition, one should note that

224 rapid evolution does not always stabilize temporal fluctuations (Becks et al. 2010; Cortez \& Patel

225 2017), in which case rapid evolution may result in priority effects via positive frequency-

226 dependence in community dynamics (Ke \& Letten 2018). Therefore, using phenotypically plastic

227 species may be more feasible for showing coexistence driven by rapid adaptive trait dynamics via

228 relative nonlinearity because phenotypic plasticity tends to strongly stabilize population

229 dynamics, and the speed of adaptation will not be as slow as genetic evolution in small 
230 populations (Yamamichi et al. 2011; Yamamichi et al. 2019).

\section{Conclusion}

233 With increasing evidence for rapid contemporary evolution on ecological timescales (Hendry

234 2016), an interest in developing a broader theory of species coexistence that incorporates rapid

235 evolution and eco-evolutionary feedbacks has grown (Tachikawa 2008; Kremer \& Klausmeier

236 2013; Wittmann \& Fukami 2018; Hart et al. 2019; van Velzen 2020; Yamamichi et al. 2020). By

237 allowing for rapid evolution in mechanistic resource competition models, we have identified a

238 new solution to the paradox of the plankton, deriving from relative nonlinearity of competition.

239 This mechanism may be important in competition between sexual and asexual species or species

240 with different mutation rates. Alongside recent empirical work demonstrating relative

241 nonlinearity across diverse systems (Letten et al. 2018; Hallett et al. 2019; Zepeda \& Martorell

242 2019), we hope that our findings will help to motivate a reappraisal of the perceived importance

243 of relative nonlinearity in nature. 


\section{ACKNOWLEDGEMENTS}

247 We thank S. P. Ellner, T. Fukami, and three anonymous reviewers for their helpful comments.

248 MY thanks N. Shinohara for stimulating discussions during the initial stages of this project. MY

249 was supported by the Japan Society for the Promotion of Science (JSPS) Grant-in-Aid for

250 Scientific Research (KAKENHI) $18 \mathrm{H} 02509$ and 19K16223.

\section{CONFLICT OF INTEREST}

253 The author declares no competing financial interests.

\section{REFERENCES}

2561.

257 Abrams, P.A. (2001). Modelling the adaptive dynamics of traits involved in inter- and intraspecific 258 interactions: An assessment of three methods. Ecol. Lett., 4, 166-175.

2592.

260 Abrams, P.A., Brassil, C.E. \& Holt, R.D. (2003). Dynamics and responses to mortality rates of competing predators undergoing predator-prey cycles. Theor. Popul. Biol., 64, 163-176.

2623.

263 Abrams, P.A. \& Holt, R.D. (2002). The impact of consumer-resource cycles on the coexistence of competing consumers. Theor. Popul. Biol., 62, 281-295.

2654.

266 Adler, P.B., HilleRisLambers, J., Kyriakidis, P.C., Guan, Q. \& Levine, J.M. (2006). Climate Sci. U.S.A., 103, 12793-12798. 
5.

270 Angert, A.L., Huxman, T.E., Chesson, P. \& Venable, D.L. (2009). Functional tradeoffs determine species coexistence via the storage effect. Proc. Natl. Acad. Sci. U.S.A., 106, 11641-11645. 6.

273 Armstrong, R.A. \& McGehee, R. (1980). Competitive exclusion. Am. Nat., 115, 151-170.

2747.

275 Barabás, G., D'Andrea, R. \& Stump, S.M. (2018). Chesson's coexistence theory. Ecol. Monogr., 88, 277-303.

2778 .

278 Becks, L., Ellner, S.P., Jones, L.E. \& Hairston, N.G., Jr. (2010). Reduction of adaptive genetic diversity radically alters eco-evolutionary community dynamics. Ecol. Lett., 13, 989-997. 9.

Bell, G. (2017). Evolutionary rescue. Annu. Rev. Ecol. Evol. Syst., 48, 605-627.

28210.

Chase, J.M. \& Leibold, M.A. (2003). Ecological Niches: Linking Classical and Contemporary

11.

Chesson, P. (1994). Multispecies competition in variable environments. Theor. Popul. Biol., 45, 227-276.

12.

Chesson, P. (2000). Mechanisms of maintenance of species diversity. Annu. Rev. Ecol. Syst., 31,

13.

Cortez, M.H. \& Patel, S. (2017). The effects of predator evolution and genetic variation on predator-prey population-level dynamics. Bull. Math. Biol., 79, 1510-1538.

14.

295 Ellner, S.P., Snyder, R.E., Adler, P.B. \& Hooker, G. (2019). An expanded modern coexistence theory for empirical applications. Ecol. Lett., 22, 3-18. 15.

298 Germain, R.M., Srivastava, D. \& Angert, A.L. (2020). Evolution of an inferior competitor increases resistance to biological invasion. Nat. Ecol. Evol., 4, 419-425. 
301 Grover, J.P. (1990). Resource competition in a variable environment: phytoplankton growing according to Monod's model. Am. Nat., 136, 771-789.

17.

Hallett, L.M., Shoemaker, L.G., White, C.T. \& Suding, K.N. (2019). Rainfall variability maintains grass-forb species coexistence. Ecol. Lett., 22, 1658-1667.

18.

307 Hart, S.P., Turcotte, M.M. \& Levine, J.M. (2019). Effects of rapid evolution on species coexistence.

19.

310 Hendry, A.P. (2016). Eco-evolutionary Dynamics. Princeton University Press, Princeton, NJ.

31120.

312 Hiltunen, T., Kaitala, V., Laakso, J. \& Becks, L. (2017). Evolutionary contribution to coexistence of competitors in microbial food webs. Proc. R. Soc. B, 284, 20170415.

31421.

315 Holt, R.D. (1977). Predation, apparent competition, and the structure of prey communities. Theor.

22.

Holt, R.D., Grover, J. \& Tilman, D. (1994). Simple rules for interspecific dominance in systems with exploitative and apparent competition. Am. Nat., 144, 741-771. 23.

Hsu, S.B., Hubbell, S.P. \& Waltman, P. (1978). Competing predators. SIAM Journal on Applied Mathematics, 35, 617-625.

24.

324 Hutchinson, G.E. (1961). The paradox of the plankton. Am. Nat., 95, 137-145.

32525.

326 Ke, P.-J. \& Letten, A.D. (2018). Coexistence theory and the frequency-dependence of priority effects. Nat. Ecol. Evol., 2, 1691-1695.

32826.

329 Kondoh, M. (2003). Foraging adaptation and the relationship between food-web complexity and stability. Science, 299, 1388-1391.

33127.

332 Kremer, C.T. \& Klausmeier, C.A. (2013). Coexistence in a variable environment: eco-evolutionary 
28.

335 Lankau, R.A. (2011). Rapid evolutionary change and the coexistence of species. Annu. Rev. Ecol. Evol. Syst., 42, 335-354.

29.

338 Letten, A.D., Dhami, M.K., Ke, P.-J. \& Fukami, T. (2018). Species coexistence through simultaneous fluctuation-dependent mechanisms. Proc. Natl. Acad. Sci. U.S.A., 115, 67456750.

30.

342 Levins, R. (1979). Coexistence in a variable environment. Am. Nat., 114, 765-783.

34331.

344 Mougi, A. (2013). Allelopathic adaptation can cause competitive coexistence. Theor. Ecol., 6, 165171.

32.

Nakazawa, T., Ohgushi, T. \& Yamamura, N. (2009). Resource-dependent reproductive adjustment and the stability of consumer-resource dynamics. Popul. Ecol., 51, 105-113.

33.

Rosenzweig, M.L. \& MacArthur, R.H. (1963). Graphical representation and stability conditions of predator-prey interactions. Am. Nat., 97, 209-223.

34.

Tachikawa, M. (2008). Fluctuation induces evolutionary branching in a mathematical model of ecosystems. PLOS ONE, 3, e3925.

35.

Tan, Z.X., Koh, J.M., Koonin, E.V. \& Cheong, K.H. (2020). Predator dormancy is a stable adaptive

36.

359 van Velzen, E. (2020). Predator coexistence through emergent fitness equalization. Ecology, 101, e02995.

36137.

362 Vasseur, D.A., Amarasekare, P., Rudolf, V.H.W. \& Levine, J.M. (2011). Eco-evolutionary dynamics enable coexistence via neighbor-dependent selection. Am. Nat., 178, E96-E109.

36438. 
365 Verdolin, J.L. (2006). Meta-analysis of foraging and predation risk trade-offs in terrestrial systems. Behav. Ecol. Sociobiol., 60, 457-464. 39.

Wilson, J.B. (1990). Mechanisms of species coexistence: Twelve explanations for Hutchinson's 'Paradox of the Plankton': Evidence from New Zealand plant communities. N. Z. J. Ecol.,

40.

Wittmann, M.J. \& Fukami, T. (2018). Eco-evolutionary buffering: Rapid evolution facilitates regional species coexistence despite local priority effects. Am. Nat., 191, E171-E184. 41.

Xiao, X. \& Fussmann, G.F. (2013). Armstrong-McGehee mechanism revisited: Competitive exclusion and coexistence of nonlinear consumers. J. Theor. Biol., 339, 26-35.

Yamamichi, M., Klauschies, T., Miner, B.E. \& van Velzen, E. (2019). Modelling inducible defences

43.

Yamamichi, M., Kyogoku, D., Iritani, R., Kobayashi, K., Takahashi, Y., Tsurui-Sato, K. et al. (2020). Intraspecific adaptation load: a mechanism for species coexistence. Trends Ecol. Evol., 35, 897-907.

44.

Yamamichi, M., Yoshida, T. \& Sasaki, A. (2011). Comparing the effects of rapid evolution and phenotypic plasticity on predator-prey dynamics. Am. Nat., 178, 287-304. 45.

Yoshida, T., Jones, L.E., Ellner, S.P., Fussmann, G.F. \& Hairston, N.G., Jr. (2003). Rapid evolution drives ecological dynamics in a predator-prey system. Nature, 424, 303-306.

392 Yuan, C. \& Chesson, P. (2015). The relative importance of relative nonlinearity and the storage effect in the lottery model. Theor. Popul. Biol., 105, 39-52. 47.

Zepeda, V. \& Martorell, C. (2019). Fluctuation-independent niche differentiation and relative nonlinearity drive coexistence in a species-rich grassland. Ecology, 100, e02726. 
a

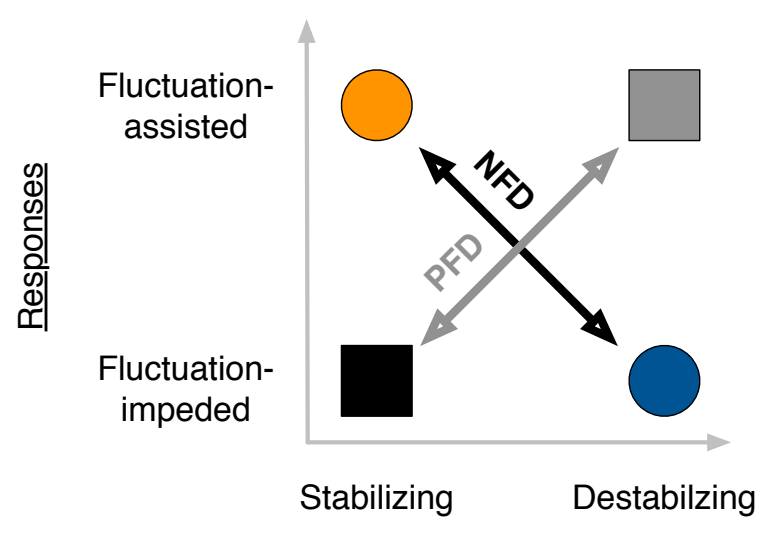

Impacts

C

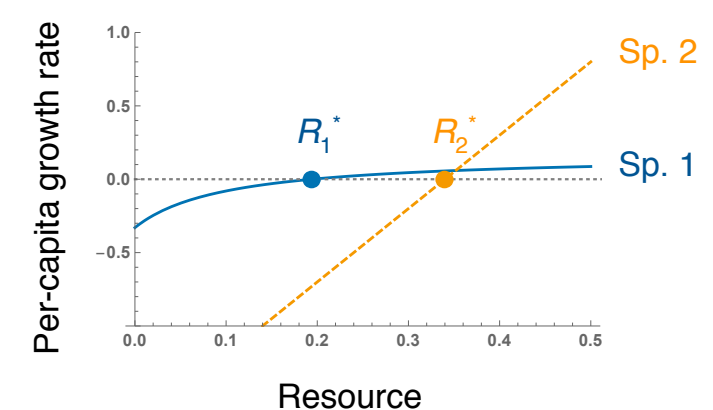

b

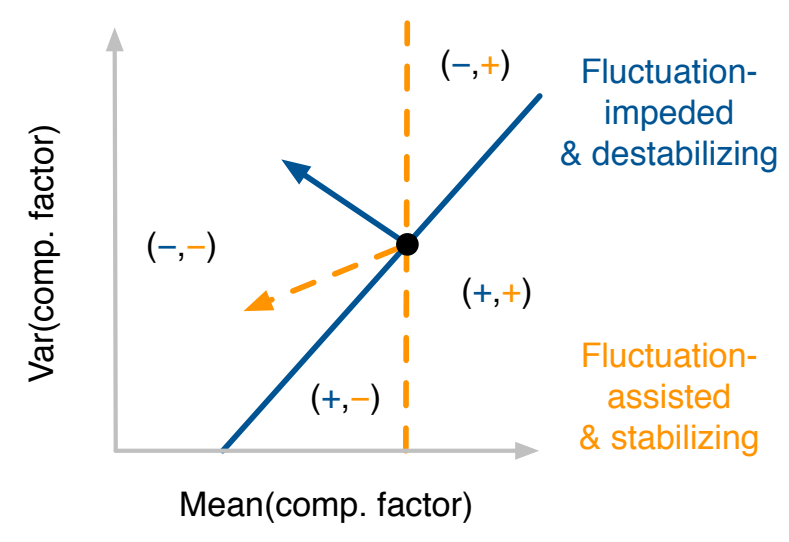

d

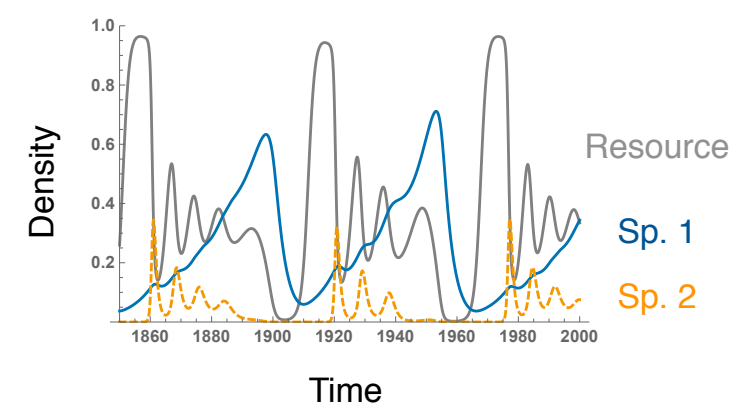

398 Figure 1 | Species coexistence by relative nonlinearity. a, Relative nonlinearity promotes coexistence with negative frequency-dependence (NFD) when one species stabilizes population

400 cycles but benefits from fluctuating environments in competition (fluctuation-assisted), while the

401 other species destabilizes dynamics but benefits from stable environments in competition

402 (fluctuation-impeded). Hence, we need to consider two aspects of the ecological niche: responses

403 and impacts (the Grinnellian and Eltonian niches, respectively: note that with a feedback cycle,

404 the ideas of response and effect are complicated since the two terms rely heavily on perspective) 
405 (Chase \& Leibold 2003). Priority effects due to positive frequency-dependence (PFD) can arise

406 when the fluctuation-impeded species stabilizes fluctuations while the fluctuation-assisted species

407 drive fluctuations (Ke \& Letten 2018). b, The concept of relative nonlinearity can also be

408 expressed in terms of zero net growth isoclines (ZNGI) (Chase \& Leibold 2003). The fluctuation-

409 assisted (fluctuation-impeded) species has higher growth rates when the variance in the

410 competitive factor (e.g., resource) is high (low), but when abundant reduces (increases) the

411 variance as shown by the impact vector. c-d, Coexistence of species with linear and saturating

412 functional responses (Abrams et al. 2003). Species 1 with a saturating functional response (blue

413 line) has a smaller $R^{*}$ and causes limit cycles with the resource (grey line), which is

414 disadvantageous due to nonlinear averaging. Species 2 with a linear functional response (the

415 dashed orange line) has a larger $R^{*}$ and stabilizes dynamics. Parameter values are $a_{1}=a_{2}=5, b_{1}=$

$416 \quad 10, b_{2}=0, d_{1}=0.33$, and $d_{2}=1.7$. 


\section{a}

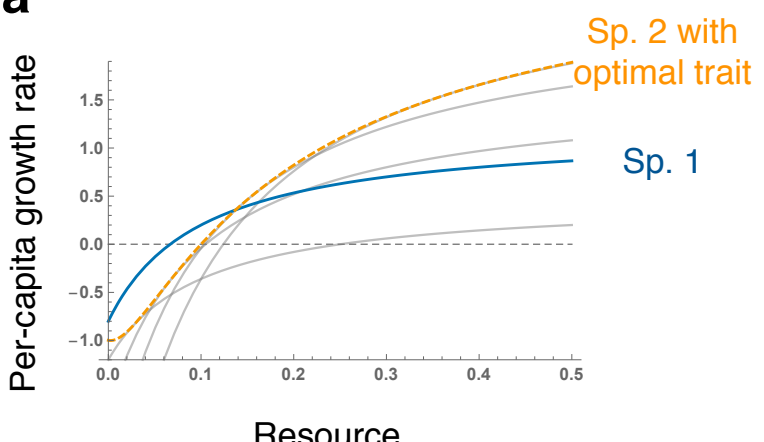

C

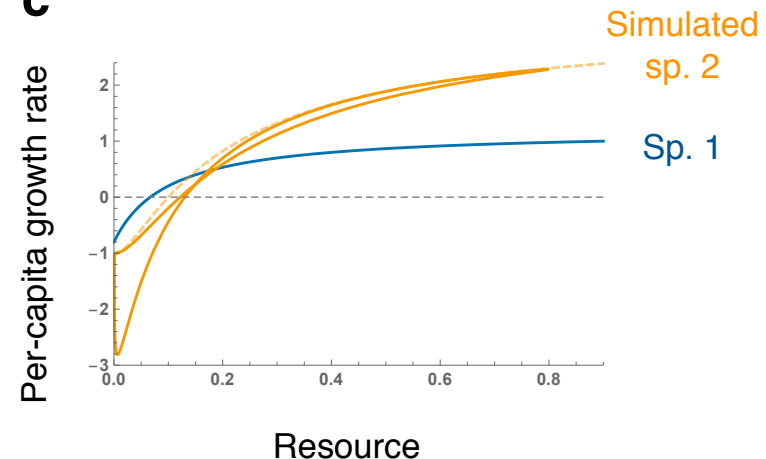

e

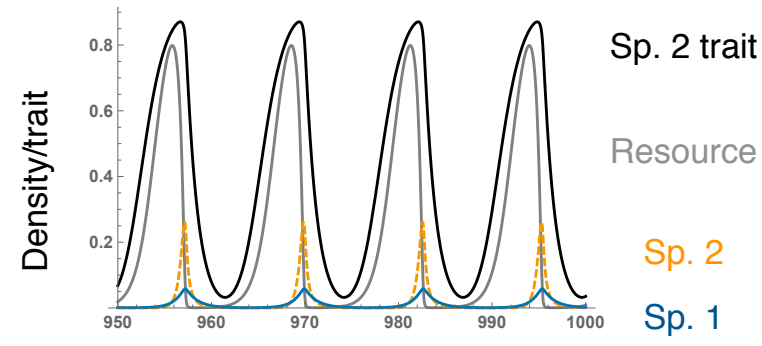

Time b

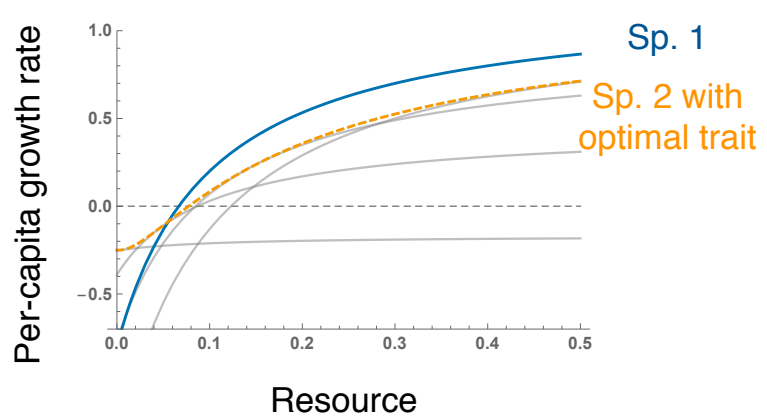

d

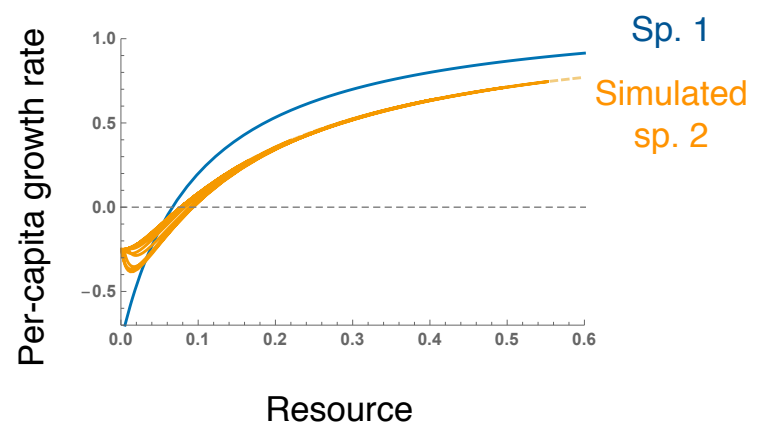

f

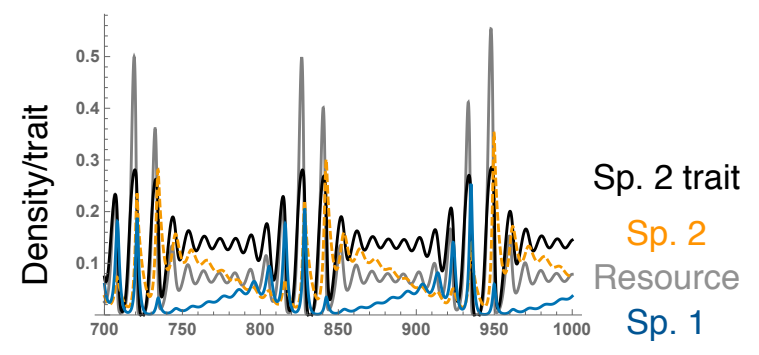

Time

419 by relative nonlinearity. a-b, The per-capita growth rate of species 2 with the optimal trait

420 (orange dashed line) is larger than that of species 1 (solid blue line) when the resource is

421 abundant (a) or scarce (b). When the trait value is fixed (gray lines), species 2 is always 
422 competitively inferior (Fig. S2). c-d, The realized per-capita growth rate of species 2 (i.e., the

423 trajectory over time: orange solid line) is different from that with the optimal trait (orange dashed

424 line) because of a time lag in adaptive evolution. e-f, Rapid evolution of species 2 results in

425 coexistence with synchronous (e) or intermittent (f) cycles. Under intermittent cycles, the density

426 of species 1 (blue solid line) increases when cycle amplitudes are small, whereas the density of

427 species 2 (orange dashed line) increases when cycle amplitudes are large. The trait value of

428 species 2 is depicted in black, and resource abundance in gray. Parameter values are $a_{1}=20, \alpha_{2}=$

$42984, b_{1}=b_{2}=10, d_{1}=0.8, \delta_{02}=1, \delta_{12}=0.2, \delta_{22}=4, V_{1}=0$, and $V_{2}=0.1$ in a, c, and e, and $\delta_{02}=$ 
a

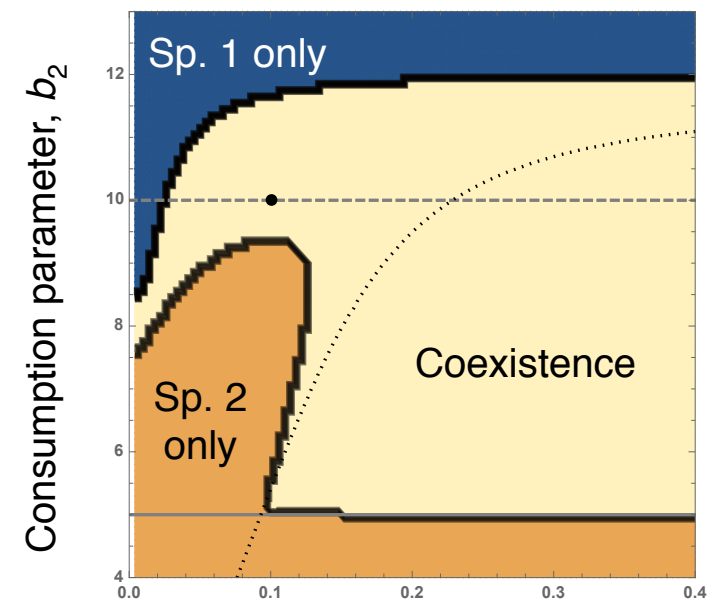

Additive genetic variance, $V_{2}$ b

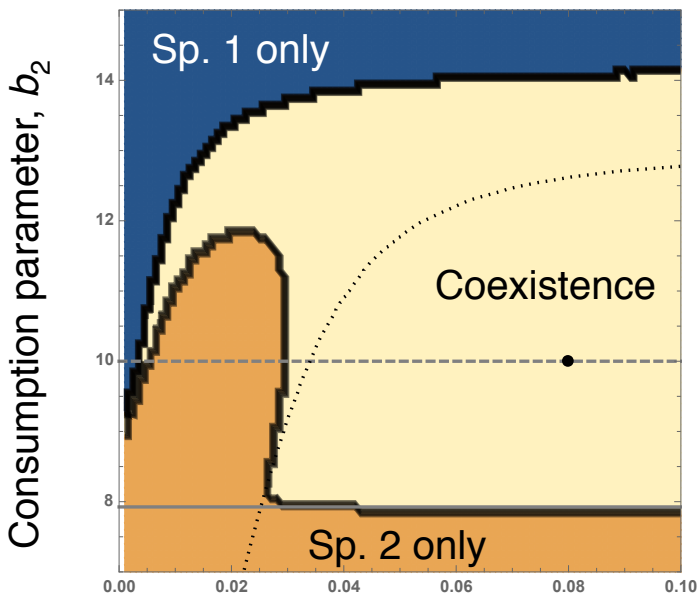

Additive genetic variance, $V_{2}$

432 Figure 3 | Effect of rapid evolution on parameter space yielding stable coexistence. a-b,

433 Phase diagrams for the genetic variance, $V_{2}$, and parameter that reduces the maximum

434 consumption rate, $b_{2}$, of species 2 where the per-capita growth rate of species 2 with the optimal

435 trait is either larger than that of species 1 when the resource is abundant (a) or scarce (b). Dashed

436 grey line indicates the situations where both species have the identical value of $b\left(b_{1}=b_{2}\right)$, where

437 species 1 is competitively superior (lower $R^{*}$ ) and therefore excludes species 2 in the absence of

438 genetic variance (Fig. 2, S2). At the limit of zero genetic variance, the coexistence region in a

439 corresponds to classical relative nonlinearity. With increasing genetic variance, the parameter

440 space corresponding to coexistence expands. Solid grey line indicates the point at which species 2

441 becomes the superior competitor and therefore genetic variance (in species 2) no longer promotes 
442 coexistence with smaller $b_{2}$. Black dots indicate fixed parameter values in Figure 2. Black dotted

443 curves indicate Hopf bifurcation in the system with species 2 and resource: the system is locally

444 stable below the curves (with smaller $b_{2}$ and larger $V_{2}$ ). 